\title{
The UML modeling elements analysis
}

\author{
Jian Zhong \\ Tianhe College Of Guangdong Polytechnic Normal University,Guangzhou,510540,China
}

\begin{abstract}
The UML modeling technology is a kind of common graphical modeling language in the object-oriented development, which is in good graces of a majority of technicians for its well-defined, expressive, powerful and widely-used feature. With the development of the society and the progress of various mechanical equipments, computer's operating instruction and language also needs updating, otherwise, it is unable for computer technicians to speed up the work efficiency in practical work.

From an objective point of view, computer technicians begin with and subject a technology or a certain demand to realize the improvement of technical level with more advanced technology or processing language integrated in the process of language development and optimization. Among all the current technologies, UML modeling technology is one of the most commonly used, which can not only objectively complete the demand of technicians, but also subjectively promote the efficiency of work at the same time. In the future, we should further strengthen the element analysis of UML modeling technology, perfect the system of technology application and promote the steady improvement of all the work.
\end{abstract}

Keywords : UML modeling technology; Element; Analysis

\section{INTRODUCTION}

The UML modeling technology, as a good language tool, since 1997 , has been highly appreciated and advocated by numerous manufacturers, organizations, experts and scholars because the various diagrams with different functions it provides, such as case-used diagram, state diagram, activity diagram and class diagram, basically cover all aspects of software engineering. In the face of the demand of current society, the application of UML modeling technology has become a trend. In the actual work, it has established a new system of high and stable working efficiency, which has a positive significance for the future development. This paper mainly analyzes the elements of UML modeling technology.

In the face of demand of actual work, the improvement only in the basic definition of UML modeling technique cannot achieve the desired results. Therefore, technicians should proceed from the reality, strengthen the practical function of UML modeling technology, prompt the widespread use by more companies and create more real value. Simply speaking, as a kind of outstanding modeling language, UML modeling technology aims to promote the communication between system developers and users, reduce the contradiction and conflict and achieve better development.

After summary and analysis, it is found that the function of the UML modeling technology mainly concentrates in the following aspects:

Firstly, the UML modeling technology can establish an ideal visualized model for the production of software system. The software systems in the past are unable to get an ideal visualized model. After the application of UML modeling technology, the visualized model can directly show the advantages and disadvantages of software, which is easy to continue improvement and development.

Secondly, the application of UML modeling technology can specify software system output, which easy to meet the actual production needs.

Thirdly, in the actual work, after the application of UML modeling technology, it needs to do important analysis, design and implementation specification in the defining process of software system redevelopment, and 
demand of all aspects is detailed, comprehensive and implementable. At the same time, the technology also makes the model established be more accurate and unambiguous.

Fourthly, UML modeling technology structures the output of software system, reduces the complex operation and promotes the the work efficiency.

Fifthly, the application of UML modeling technology can establish a wholesome document for the output of software system, so that all software systems can be provided with their backup information.

Lastly, UML modeling technology can establish corresponding documents for system architecture and all the detailed parts. From the perspective of the features of the UML modeling technology, it is more comprehensive on the whole and can guarantee for the necessity of the actual work.

\section{UNIFIED MODELING LANGUAGE OF UML MODELING TECHNOLOGY}

\subsection{Semantics}

As the deepening of the daily work difficulty, simple technology has been unable to meet the requirements of actual work. The old software cannot meet the requirements and even affect the progress of the work so as to cause economic loss to enterprises in the product design and graphic processing. Therefore, with technician's long-term research and optimization, the wholesome UML modeling technology is finally established.

One of the advantages of this technology is that it has unified modeling language (UML). The actual application of UML modeling technology is relatively smooth, so there won't be complete operation.

In general, unified modeling language of UML modeling technology consists of two parts: semantics and notation. Conceptually, the semantics of UML modeling technology mainly describes the accurate definition of meta-model. All elements in meta-model are provided with a simple, consistent and commonly used definitional instruction. Through the definitional instruction, UML modeling technology can help developers achieve a high consistency in semantics and objectively eliminates the harmful effects caused by the best representation methods varying from person to person. So, in the further application process of UML modeling technology, we should strengthen the analysis and application of semantics.

\subsection{Notation}

As an important part of UML modeling technology, notation has a great effect on the practical application of UML modeling technology. In the practical application, the notation of UML modeling technology is mainly used to define symbols, and at the same time, provides more standard for the developer or a development tool, including graphic symbol and text grammar standard, etc. With the reference of standards, actual effect of UML modeling technology will be more prominent, which improves the efficiency of all the work and optimizes economic benefit to a great extent.

It is important to note that the important part of the UML modeling technology also can be expressed with five diagrams.

The first kind is to case-used diagram, which describes the system function from the user's perspective and points out that the operator of each function.

The second kind is static diagram, including class diagram, object diagram and package diagram.

The third kind is the behavior diagrams, including state diagram and activity diagram, which is used to describe the interactive relationship between the system dynamic model and the component object.

The fourth kind is interaction diagram, including the sequence diagram and collaboration diagram (also known as interaction diagram), which is used to describe the interaction relationship between objects.

The fifth kind is implementation diagram, including component diagram and configuration diagram. 


\section{APPLICATION AREA OF UML MODELING TECHNOLOGY}

UML modeling technology, as one of the most advanced technologies, must ensure the diversity of function in practical application. At present, the problem has been breached and ideal results are obtained.

However, to create more economic value by means of UML modeling technology, we must broaden the application field of UML modeling technology and prompt it to provide service for more fields, namely, realize the user's universality.

After a lot of discussion and research, it is thought that UML targets to describe any type of system based on an object-oriented way, which has a wide application field. One of the most commonly used field is to establish model of software system, but it can also be used to describe the non-software field, such as mechanical system, enterprise institution or business process, industrial system or industrial process with real-time requirements, etc In addition, it is also applicable to the different stages from requirements specification description to system testing completion in the system development process. In a word, UML is a standard modeling language, which can be used in the system modeling process of any static structure and dynamic behavior.

\section{ELEMENTS OF UML MODELING TECHNOLOY}

\subsection{UML Modeling technology}

UML modeling technology is to establish the model of the whole system with modeling elements, therefore, it can be restructured and applied according to user's requirement.

At present, the modeling elements of the applied UML modeling technology are relatively more, so, there is a complicated relationship in the process of system modeling.

For example, the modeling elements of UML modeling technology consist of the internal class relations. In the actual use of UML modeling technology, to better meet the demand of users, UML modeling technology provides a variety of description model elements with graphical visualization, that is to say, in UML modeling technology, the same model element may appear in more than one graphs and may correspond to multiple graphical elements. With its multiple types and methods in the collocation, users can investigate models more effectively from different perspectives in order to get ideal results.

In general, the applied UML modeling technology can be divided into structure modeling and dynamic modeling. The structure modeling is to describe the system statically from the internal structure, which is applicable in static diagram, case-used diagram, implementation and configuration diagrams by adopting class diagram, case-used diagram, component diagram and configuration diagrams and other graphs. Dynamic modeling is to describe the system from the interactions and message passing between the dynamic behavior and composite object, which is applicable in state machine diagram, activity diagram, sequence diagram and collaboration diagram, etc. UML modeling technology, with its comprehensive function and diversified composition, is able to complete the more projects in the practical work, which objectively promotes the efficiency of work.

\subsection{Purpose and Principle of UML Modeling technology}

In the research process of UML modeling technology, it needs to consider the final service condition. Therefore, from the perspective of technical elements analysis, purpose and principle of UML modeling technology has a great influence on the further development and perfection. At this point, this paper analyzes the purpose and principle of UML modeling technology in details.

First, the purpose of the UML modeling technology development is to help project designer to meet objective demand.

Second, in the application process of UML modeling technology, the technology should provide detailed method of system structure or behavior. Due to the 
diversity of technology type at present, the user's choice is more extensive, therefore, UML modeling technology should not only provide users with detailed method, but also meet their wide demands.

Third, the application of UML modeling technology in daily work can give a guidance model for system construction, which can be used as the foundation to realize the diversification of structure and improve work efficiency.

Fourth, the application of UML modeling technology should document the decisions made. From the above statement, the purpose of this technology is quite clear.

In principle, the technology's requirement is simpler, for example, what models UML modeling technology prefer to create has a far-reaching influence on how to solve the problem and solution; Each kind of model can be expressed in different precision level; The best model is associated with reality; A single model is inadequate and it is better to deal with an important system with a group of independent models.

\subsection{Suitable Targets of UML modeling technology}

The service areas of UML modeling technology is wide and function of it is diverse, but different users need different functions of UML modeling technology. Therefore, the suitable targets of UML modeling technology have specificity.

Firstly, in terms of business modeling, most users of UML modeling technology are experts in various fields, especially demand analysts, at the same time, systems analysts and architects can also be suitable users of UML modeling technology to speed up the research.

Secondly, in terms of demand model, demand analysts are the main suitable users, and systems analysts can also be the main users to conduct research, and other architects or developers can also be appropriate to participate in UML modeling to deepen research and get more results.

\section{CONCLUSIONS}

With the unceasingly development of computer technology, computer language and related processing technology has also got great progress. UML modeling technology, as an outstanding computer technology, has an ideal achievement in the practical application. From an objective point of view, it has achieved great success both in function and in technical level. In the future, in-depth analysis of UML modeling technology should be conducted to strengthen the connotation of the application and accomplish more complex and difficult problems so that greater economic and social benefits can be created.

\section{REFERENCE}

[1]Yang Caixia, Liurui, Chenkui. "E-commerce Platform Analysis and Design of College Students' Creative Work". Science-Technology and Management, 2014, 03:53-58.

[2]Dai Yumei, Fengyan. "Curriculum Design of UML Modeling Technology Based on the Working Process of the Software Technology". Journal of Zhongzhou University, 2014, 02:125-128.

[3]Huang Xing. "Research on UML Modeling Strategy of Database Application System”. Electronic Technology and Software Engineering, 2014, 05 :203.

[4]Li Lvjun. "Research and Application of UML Modeling Technology Based on RUP". Computer Knowledge and Technology, 2014, 11:2556-2561.

[5]Bu Ning, Liu Yuling, Lian Yifeng, Huang Liang. “A UML Modeling Analysis Method Based on Network Security Syste". Computer Research and Development, 2014, 07:1578-1593.

[6]Chen Guangzhi, Pan Rong, Li Lei. "Review and Research Trend of Workflow Modeling Technology". Computer Science, 2014, S1:11-17+23.

[7]Wang Xiaoyan, Li Xiaojie, Shi Menglin. "Curriculum Reform and Practice of UML Modeling Technology in the Integration Mode of Production and Education". China Electric Power Education, 2014, 21:122-124. 